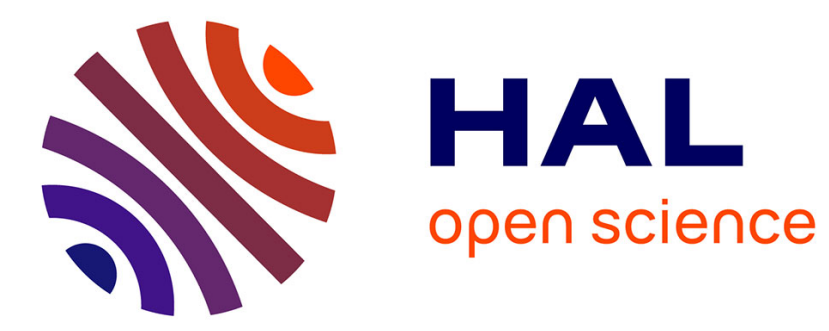

\title{
Coronary vein tracking from MSCT using a minimum cost path approach
}

\author{
Marie-Paule Garcia, Christine Toumoulin, Pascal Haigron, Jérôme Velut, \\ Mireille Garreau, Dominique Boulmier
}

\section{- To cite this version:}

Marie-Paule Garcia, Christine Toumoulin, Pascal Haigron, Jérôme Velut, Mireille Garreau, et al.. Coronary vein tracking from MSCT using a minimum cost path approach. IEEE International Symposium on Biomedical Imaging: From Nano to Macro (ISBI 2010), Apr 2010, Rotterdam, Netherlands. pp.17-20, 10.1109/ISBI.2010.5490424 . inserm-00526972

\section{HAL Id: inserm-00526972 https://www.hal.inserm.fr/inserm-00526972}

Submitted on 19 Oct 2010

HAL is a multi-disciplinary open access archive for the deposit and dissemination of scientific research documents, whether they are published or not. The documents may come from teaching and research institutions in France or abroad, or from public or private research centers.
L'archive ouverte pluridisciplinaire HAL, est destinée au dépôt et à la diffusion de documents scientifiques de niveau recherche, publiés ou non, émanant des établissements d'enseignement et de recherche français ou étrangers, des laboratoires publics ou privés. 


\title{
CORONARY VEIN TRACKING FROM MSCT USING A MINIMUM COST PATH APPROACH
}

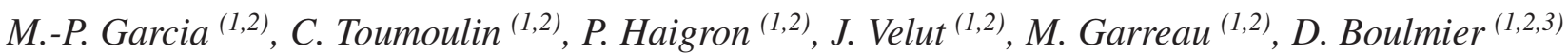 \\ (1) INSERM, U642, Rennes, F-35000, France; \\ (2) Université de Rennes 1, LTSI, Rennes, F-35000, France; \\ (3) CHU Rennes, Service de cardiologie et maladies vasculaires, Rennes, F-35000, France;
}

\begin{abstract}
In this paper, we deal with the problem of tracking the coronary venous tree from Multi-Slice Computed Tomography (MSCT) angiography. Contrast inhomogeneities are a major issue. The proposed tracking procedure is based on minimum-cost path computation and makes use of 'FastMarching' technique. The algorithm aims at propagating a front inside a vascular structure and extracting a centered path. To achieve this goal, a specific cost function which combines the vessel local orientation to a vesselness measure is designed. Experiments on synthetic data and real data have been performed. Coronary veins with contrast difficulties are extracted with a low computing time.
\end{abstract}

Index Terms - Coronary veins, Computed Tomography Angiography, Vessel tracking, Minimum cost path, Fast Marching

\section{INTRODUCTION}

Cardiac resynchronization therapy (CRT) is assessed as an efficient therapeutic approach for patients who suffer from severe heart failure, sinus rhythm and ventricular conduction delay [1]. The procedure consists in pacing simultaneously or with a small delay, both the right ventricle (RV) and the left ventricle (LV). Daubert and al. proposed a totally transvenous approach [2]. LV lead is placed through the coronary venous tree which is challenging due to the difficulty in accessing some pacing sites and the risk of dissecting the coronary sinus. In order to improve and secure the implant procedure, the objective is to describe patient's coronary venous anatomy and provide cardiologists with a CRT planning. In this paper, the study is carried out using contrast-enhanced Multi-Slice Computed Tomography (MSCT) scans. Due to the cardiac circulatory system, the dye product passes first through the coronary arteries and then is gathered by the coronary veins. Once the venous tree is reached, its distribution is non-uniform and attenuated leading to variable densities along the tree, hypo-dense zones, inhomogeneous contrast

\footnotetext{
Acknowledgments: This work is supported by the French Research Minister (n 04 T 187 - 188 - 189 - 190 CITH and n ANR-07-EMPB-007-01) and the Brittany region.
}

filling, blurred contours and attachment of nearby structures (cavities, arteries...).

In the literature, many vessel extraction techniques are proposed. We first studied a sequential axis tracing approach based on geometrical moments [3,4]. In this paper, we focused on minimum-cost path approaches which appear less sensitive to noise perturbation. Minimal path computation relies on a cost function definition which assigns a value to each node in a graph representation of the image. This definition has to fit the features of the curve of interest. Definitions based on the image intensity information appear robust for structures with homogeneous contrast [5]. When dealing with structures with non-uniform contrast, vesselness measures based on eigenvalue analysis of the Hessian matrix yield successful results [6]. An efficient and quick way to solve the minimal path problem in 3D images is the 'FastMarching' procedure. Our method is based in part on the approach proposed by Cohen and Deschamps [7], where the 'Fast-Marching' algorithm is used to extract elongated tubular structures. Considering the combination between orientation information and Frangi's vesselness measure [8], we propose in this paper a new specific cost function for coronary veins in MSCT images.

This paper is organized as follows. In Section 2, we first make a review of the 'Fast-Marching' algorithm and the multiscale vessel filtering technique using the Hessian matrix. We then develop our vessel tracking procedure in Section 3. Finally, results are presented in Section 4 and discussed in Section 5.

\section{BACKGROUND}

\subsection{Fast-Marching and Minimum-Cost Path}

The Fast-Marching algorithm is based on the well-known Dijkstra algorithm(1959) which considers the image as a grid of nodes, where each node corresponds to an image voxel. A cost function value is assigned to every node. The shortest path between two image voxels becomes the one that has the lowest cumulative cost between the two corresponding nodes, among any other paths between the same two nodes. Given a cost function $\Lambda$ taking lower values near the desired fea- 
tures and a seed point $P_{0}$, the minimal path $C(s)$ from $P_{0}$ to any point $P_{x, y, z}$ corresponds to the path along which the cumulative travel cost is minimal. The minimal travel cost is expressed as follows :

$$
U_{x, y, z}=\min _{C} \int_{P_{0}}^{(x, y, z)} \Lambda(C(s)) d s
$$

The 'Fast-Marching' algorithm describes a front propagation scenario, where the value of $U_{x, y, z}$ is the time $t$ at which the front passes over the point $P_{x, y, z}$ with a front speed $1 / \Lambda_{x, y, z}$. The arrival time (cumulative travel cost) $U_{x, y, z}$ is computed thanks to the Eikonal equation $|\nabla U|=\Lambda$. Once the final point $P_{F}$ is reached by the front, a back-propagation on the arrival time map $U$ is applied in order to extract the minimal cost path from $P_{0}$ to $P_{F}$.

\subsection{Vessel Filtering}

Second-order derivatives computation at multiple scales allows to characterize tubular structures. This multi-scale approach is based on the eigenvalue analysis of gray-level Hessian matrices. Indeed, the Hessian matrix can be decomposed into three eigenvalues ordered according to their magnitude as $\left|\lambda_{1}\right| \leq\left|\lambda_{2}\right| \leq\left|\lambda_{3}\right|$. For an ideal tubular structure, the smallest eigenvalue $\lambda_{1}$ is close to zero, (reflecting a minimum intensity variation). The magnitudes of the other two eigenvalues $\lambda_{2}$ and $\lambda_{3}$ are high and close to being equal. These previous hypotheses are used in Hessian-based filters in order to compute a likelihood measure for a voxel belonging to a vessel. In this work, we used the filter defined by Frangi in [9], since it yields reasonable vesselness measures in our data sets in regions that contain close proximity. For each scale, the vesselness measure $F_{x, y, z}$ at position $(x, y, z)$ is computed as:

$F_{x, y, z}=\left\{\begin{array}{l}0, \text { if } \lambda_{2}>0 \text { or } \lambda_{3}>0 \\ \left(1-\exp \left(\frac{-R_{A}^{2}}{2 \alpha^{2}}\right)\right) \exp \left(\frac{-R_{B}^{2}}{2 \beta^{2}}\right)\left(1-\exp \left(\frac{-S^{2}}{2 \gamma^{2}}\right)\right)\end{array}\right.$

with $R_{A}=\left|\frac{\lambda_{2}}{\lambda_{3}}\right|, R_{B}=\frac{\left|\lambda_{1}\right|}{\sqrt{\left|\lambda_{2} \lambda_{3}\right|}}$ and $S=\sqrt{\lambda_{1}^{2}+\lambda_{2}^{2}+\lambda_{3}^{2}}$

The parameters $\alpha, \beta$ and $\gamma$ are used to control the sensitivity of the filter to deviations from cylindrical shape. The filter $F$ is applied at different scales, and yields a maximum response at a scale approximating the vessel radius. The resulting vesselness measure is expected to be maximum at the vessel center and close to zero outside them.

\section{THE VESSEL TRACKING ALGORITHM}

This section introduces our vessel tracking algorithm designed for coronary veins from MSCT data sets. We make use of the 'Fast-Marching' algorithm, described in Section 2.1 to extract centered trajectories. The tracking procedure allows to extract a whole vein from a seed point to a final point defined interactively.

In our approach, we want the front to evolve fastest in the direction of the vessel local orientation and in regions where the vesselness measure is higher (close to the vessel axis). Furthermore, to avoid noise disturbance due to contrast problem, we do not want the front to propagate through vessel boundaries. Therefore, some constraints are applied to the front. It can only propagate into: (1) a restricted area defined by an angular value $r$ in the vessel direction and (2) vessel regions (according to Frangi's filter response).

The cost function $\Lambda$ is a non linear function which considers the reciprocal of the Frangi's vesselness measure $F_{x, y, z}$ weighted by the orientation information $\rho$, defined as:

$$
\begin{gathered}
\Lambda_{x, y, z}= \begin{cases}\left(\frac{1}{F_{x, y, z}} \cdot \rho\right)^{2} & \text { if } \theta<r \text { and } F_{x, y, z}>s \\
C, \quad & \text { otherwise }\end{cases} \\
\text { with } \rho=\sin (\theta)
\end{gathered}
$$

where $C$ is the cost given to voxels located outside the propagation area (threshold $r$ ) and also at non-vessel positions (threshold $s$ ) and $\theta$ the angle between the direction of the normal to front at position $(x, y, z)$ and the local orientation. The adopted value for $r$ is given in section 4 . The cost $C$ is an insensitive parameter, it must be high enough to stop the front evolution at considered locations $(C=10000)$. The vesselness measures $F$ are normalized with respect to the highest response. The threshold $s$ is also quite insensitive, it must be close to zero ( $s=0.01)$.

The cost function definition is efficient to keep the front propagating inside the vessel when the considered segment is close to the front head. However, the front may flow over the boundaries of tracked segments away from the head. This is the case when background or nearby structures are not detected. Indeed, the cost given to the points located on the front surface is not high enough to definitively stop the front propagation. That is to say, the previous points may be considered later on, leading to a leak. Thus, we need to stop artificially the front at those points when they are distant from the head of the front. This additional process called Freezing was first introduced in [7]. A distance information $d$ is required to define the length of the tracked segment located at front head, i.e. points that can still be considered in the propagation process.

\section{RESULTS}

\subsection{Experiments on synthetic data}

We have studied the behaviour of the proposed algorithm on a synthetic data set. An isotropic synthetic data set of size $110 \times 90 \times 90$ voxels with features similar to real data sets, 
has been designed from the VTK library. A surface representation and a curvilinear reconstruction along the main vessel axis are shown in Figure 1. For geometry simplicity, the tubular structure is based on a real coronary vein description containing a bifurcation. To cope with typical coronary vein radius, the tube radii varies from 1 to 6 voxels. Furthermore, typical cardiac structures are represented on the data set, such as a sphere for cavities and cylinders for coronary arteries. As regards the densities laying inside the tube, difficulties due to dye product have been considered. Indeed, an alternating sequence of hypo-, hyper- and iso-dense areas have been set in the range [100 - 300] Hounsfield Units (HU). The tubular structure was also smoothed using a Gaussian kernel to make blurred contours. The background was set to $-80 \mathrm{HU}$ (mean density of adipose epicardic tissue).

First of all, some tests have been performed on Frangi's filter parameters to enhance the response filter close to vessel axis and reduce noise disturbance at contour proximities. Thus, $\alpha, \beta, \gamma$ have been set to $0.5,0.5$ and 50 respectively and the scale range to $[1-4]$ voxels.

Further tests on the parameters guiding the front propagation have been carried out. The 'Fast-Marching' algorithm was applied using a 6-neighborhood. We performed some statistical measurements on the signed euclidian distances between each front surface point to the closest point of the synthetic vessel, using boxplots. The central box indicates the location of the $50 \%$ central values between the lower and upper quartile. Figure 2 presents the boxplots of the distances distribution for angular values $r$ varying from 60 to 90 degrees coupled to the Freezing distance $d$ varying from 6 to 12 voxels. Each distance distribution was computed from data sets with additive Gaussian noise of varying standard deviation $\sigma_{\text {noise }}=[0,10,20,30,40,50]$. We can observe that the angular value $r=80$ stops the front before vessel boundaries (position 0) and is not sensitive to the Freezing distance. Thus, we set $r$ to 80 degrees and $d$ to 12 voxels.

Finally, we chose to compare our cost function definition based on orientation constraint and Frangi's vesselness measure $(\mathrm{VM}+\mathrm{OC})$, with another one based only on the Frangi's vesselness measure $(\mathrm{VM})$ :

$$
\Lambda(x, y, z)= \begin{cases}\frac{1}{F_{x, y, z}} & \text { if } F_{x, y, z}>s \\ C, & \text { otherwise }\end{cases}
$$

We applied our vessel tracking algorithm with the previously fixed parameters. In Figure 3, boxplots of the distances distribution for $\sigma_{\text {noise }}$ varying from 0 to 50 are displayed. We can observe that our algorithm with orientation constraint stops generally the front before the vessel contours, whereas the method based on the Frangi's vesselness measure cannot prevent the front from flowing over the boundaries. In order to assess the centerline tracking efficiency, we used an overlap score $(\mathrm{OV})$ which represents the ability to track the whole vessel [10]. The results are introduced in Figure 4,

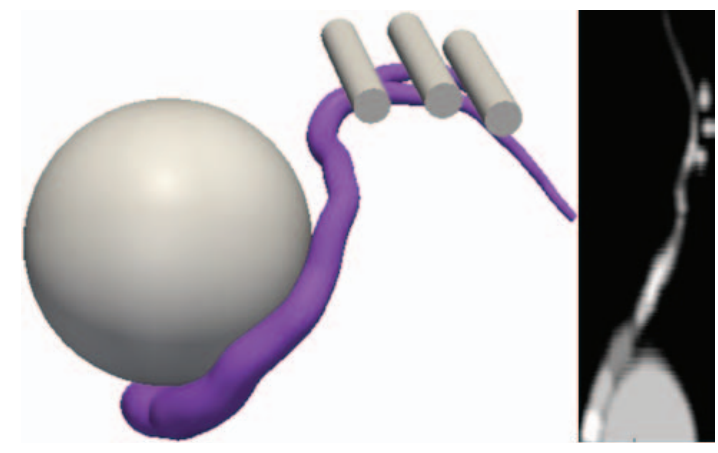

Fig. 1. Synthetic data set. Left: Surface representation. Right: Curvilinear Reconstruction along the main vessel axis.
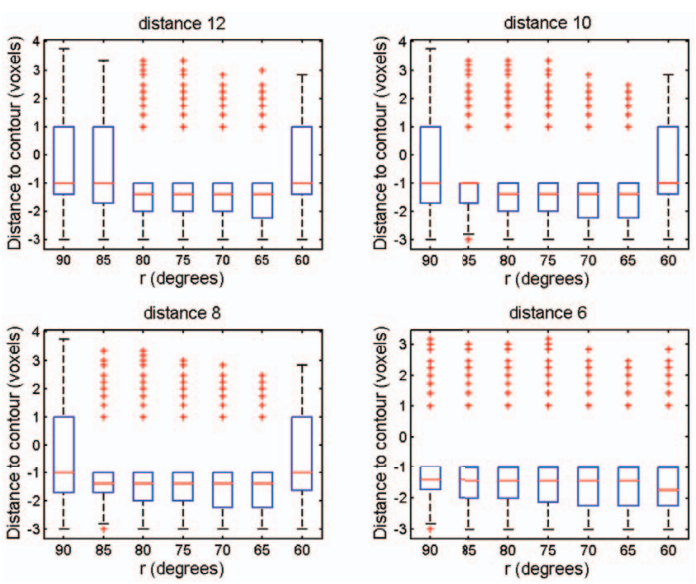

Fig. 2. Distance to the border for varying angular values $r$ and varying Freezing distance $d$.

where we can notice the sensitivity of the method based on the Frangi's vesselness measure (VM) to noise disturbance whereas our method with orientation constraint $(\mathrm{VM}+\mathrm{OC})$ yields high scores for different $\sigma_{\text {noise }}$. Furthermore, we can observe the high difference in computing time between both methods. The time computation depends on the number of points visited by the front.

\subsection{Experiments on real data}

Experiments have been carried out on three MSCT data sets acquired on a General Electric LightSpeed VCT 64-slice Scanner. The identical protocol was used for all the acquisitions. Each data set includes 20 volumes reconstructed from slices acquired every $5 \%$ of the cardiac cycle. There are approximately 200 slices per volume, with $512 \times 512$ pixels per slice. The resolution is about $0.4 \times 0.4 \mathrm{~mm}^{2}$ per pixel. A preliminary interpolation was performed to make the datasets isotropic. Coronary venous tree is better contrasted from the $50 \%$ volume to the $80 \%$ volume. A medical expert labelled the coronary venous tree on seven volumes from the three data sets and classified 38 veins according to their visibil- 

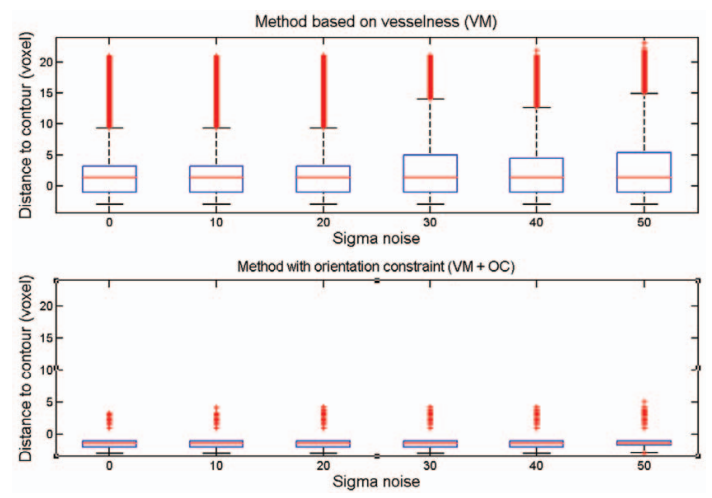

Fig. 3. Distance to the border for two different methods.
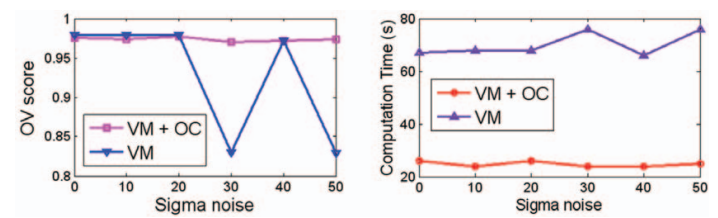

Fig. 4. Left: Overlap Score of the extracted path along the main vessel for different $\sigma_{n o i s e}$ and for two different methods. Right: Computing time.

ity (Table 1). Four categories are distinguished: high quality $(++)$, good quality $(+)$, medium quality $(+-)$ and low quality $(--)$.

As previously on synthetic data, we compared our cost function definition $(\mathrm{VM}+\mathrm{OC})$ with the one based on the Frangi's vesselness measure (VM). Considering our planning context, we evaluated the method extraction capability, i.e. its ability to track a whole vein given interactively defined seed and final points. Both methods were applied to the 38 labelled veins and the percentage of successful path extractions according to vessel quality is given in Table 2 (unsuccessful extractions did not reach the final point). The 'Fast-Marching' algorithm was applied using a 6-neighborhood. The vessel tracking algorithm was executed with the parameter values set from the synthetic data study. Our cost function definition based on orientation constraint and Frangi's vesselness measure $(\mathrm{VM}+\mathrm{OC})$ yields better results for good and medium quality vessels. This preliminary evaluation has to be confirmed on more data.

\begin{tabular}{|c|c|c|c|c|}
\hline Vessel Quality & ++ & + & +- & -- \\
\hline Coronary Sinus & 7 & 3 & 3 & $/$ \\
\hline Mid Cardiac Vein & 1 & 4 & $/$ & 1 \\
\hline Postero-Lateral Vein & 2 & 2 & 1 & 4 \\
\hline Great Cardiac Vein & $/$ & 3 & 2 & 1 \\
\hline Lateral Vein & $/$ & 2 & $/$ & 2 \\
\hline
\end{tabular}

Table 1. Labelled coronary veins and their visibility in seven MSCT volumes from three data sets.

\begin{tabular}{|c|c|c|c|c|}
\hline Vessel Quality & ++ & + & +- & -- \\
\hline Methode VM & $100 \%$ & $86 \%$ & $50 \%$ & $12 \%$ \\
\hline Methode VM + OC & $100 \%$ & $93 \%$ & $83 \%$ & $12 \%$ \\
\hline
\end{tabular}

Table 2. Percentage of successful path extractions according to vessel visibility on MSCT data sets.

\section{CONCLUSION}

A tracking algorithm was proposed to deal with coronary vein extraction difficulties due to contrast inhomogeneities in MSCT volumes. The specific cost function combined to a Freezing process yields successful results on minimal path extraction. Furthermore, the algorithm computing time is low since the front evolves only inside the vascular structures, reducing the number of visited points.

\section{REFERENCES}

[1] C. Leclercq and D.A. Kass, "Re-timing the failing heart: Principles and current clinical status of cardiac resynchronization," J. Am. Coll. Cardiol., vol. 39, no. 2, pp. 194-201, 2002.

[2] J. Daubert, P. Ritter, H. Le Breton, D. Gras, C. Leclercq, A. Lazarus, J. Mugica, P. Mabo, and S. Cazeau, "Permanent left ventricular pacing with transvenous leads inserted into the coronary veins," PACE, vol. 21, pp. 239-245, January 1998.

[3] M.-P. Garcia, C. Toumoulin, M. Garreau, C. Kulik, D. Boulmier, and C. Leclercq, "An improved spatial tracking algorithm applied to coronary veins into cardiac multi-slice computed tomography volume," in EMBS, 2008.

[4] A. Larralde, C. Boldak, M. Garreau, C. Toumoulin, D. Boulmier, and Y. Rolland, "Evaluation of a 3D segmentation software for the coronary characterization in Multi-Slice Computed Tomography," in FIMH, 2003, vol. 2674, pp. 39-51.

[5] T. Deschamps and L.D. Cohen, "Fast extraction of minimal paths in 3D images and applications to virtual endoscopy," Medical Image Analysis, vol. 5, no. 4, pp. 281-299, Dec 2001.

[6] S.D. Olabarriaga, M. Breeuwer, and W.J. Niessen, "Minimum Cost Path Algorithm for Coronary Artery Central Axis Tracking in CT Images," in MICCAI (2), 2003, pp. 687-694.

[7] L.D. Cohen and T. Deschamps, "Segmentation of 3D tubular objects with adaptive front propagation and minimal tree extraction for 3D medical imaging," Computer Methods in Biomechanics and Biomedical Engineering, vol. 10, no. 4, pp. 289-305, August 2007.

[8] M. Jackowski, X. Papademetris, L.W. Dobrucki, A.J. Sinusas, and L.H. Staib, "Characterizing Vascular Connectivity from microCT Images," in MICCAI (2), 2005, pp. 701-708.

[9] A.F. Frangi, W.J. Niessen, K.L. Vincken, and M.A. Viergever, "Muliscale vessel enhancement filtering," in MICCAI, 1998, pp. 130-137.

[10] C.T. Metz, M. Schaap, T. van Walsum, A.G. van der Giessen, A.C. Weustink, N.R. Mollet, G. Krestin, and W.J. Niessen, "3D Segmentation in the Clinic: a Grand Challenge II - Coronary Artery Tracking," in MICCAI, 2008. 